\title{
Evaluation of the combined antibiotics exposure using the simulator of human intestinal microbial ecosystem (SHIME)
}

Lei Liu

Nankai University

Hongmei Qi

Nankai University

Pengcheng Suo

Nanjing University of Information Science and Technology

Huai Lin

Nankai University

Siyi Wang

Nankai University

Xiaojun Zuo ( $\nabla$ z850108xj@163.com )

Nanjing University of Information Science and Technology https://orcid.org/0000-0001-6823-1962

Research

Keywords: Amoxicillin (Amx), Bifidobacterium longum, Gentamycin (Gen), Inulin, Simulator of the human intestinal microbial ecosystem (SHIME)

Posted Date: August 17th, 2020

DOl: https://doi.org/10.21203/rs.3.rs-46837/v1

License: (c) (1) This work is licensed under a Creative Commons Attribution 4.0 International License.

Read Full License 


\section{Abstract}

Background: Antibiotics are emerging toxic contaminant that have potential public health risk worldwide, which also would cause human intestinal microbial disorder and develop multiple human diseases. However, to date, the combination effects of antibiotics on human intestinal microbiota dysbiosis and related health risk are not fully understood. Moreover, there is limited information on using probiotics or synbiotics for restoration of intestinal microbiome affected by antibiotics. Therefore, this study evaluated the in vitro ability of combined effects of amoxicillin (Amx) and gentamycin (Gen), and the restoration effects of probiotics or synbiotics on ARGs as well as human disease-related pathways in the simulated human gut.

Results: This study indicated that the combination exposure of Amx and Gen was confirmed to promote the increase of most ARGs and the disease-related pathways, which may be better restored by probiotics treatment. The results of the alpha diversity of the combined antibiotics exposure or the recovery microbial community showed no difference from the control. However, the beta diversity results indicated their differences, and the ascending colon sample recovered better under natural condition while the descending colon sample recovered better after probiotics treatment. Combination effects on the genetic level might attribute to microbiota shift, which were explained well by the phenomenon that Escherichia/Shigella was positively associated with the ARGs, and Klebsiella and Escherichia/Shigella were positively related to the human disease-related pathways.

Conclusion: These results might be valuable to direct the future work and opened up new perspectives to address the direct effects of combine antibiotics on the intestinal microbiota and find a promising strategy to restore the antibiotics associated dysbiosis of gut microbiota.

\section{Background}

The antibiotic therapies have been demonstrated paramount importance in the treatment of bacterial infections since its discovery in the 1940s. Nowadays the extensively used antibiotics are considered as toxic emerging contaminants, which pose severe threats to environmental ecosystems [1]. Humans are directly and indirectly exposed to different antibiotics cocktail through inhalation, ingestion of drugs, drinking water, and foods, and their risk assessments have attracted more attention, recently [2-4]. As a result, antibiotics may progressively enter into the human gastrointestinal tract and produce a large variety of antibiotic-resistant gut-micro flora [5]. In our previous research, it was found that both human and veterinary antibiotics were mostly detected in the gut of the Chinese population [6]. The stable intestinal microbial ecosystem has been demonstrated not only to provide essential nutrients for human health but also to modulate the immune function by protecting infectious pathogens $[7,8]$. However, exposure to antibiotics cocktail may lead to the disrupt of the stable ecosystem and promote the spread of antibiotic-resistant bacteria (ARB) and antibiotic resistance genes (ARGs) in the human gut, which may limit the treatment efficiency and resulting from the chronic and relapsing infectious diseases [9-11]. 
Amoxicillin (Amx) is a broad-spectrum oral penicillin-type beta-lactam antibiotic, which kills bacteria by interfering with the synthesis of bacterial cell wall peptidoglycan layers $[12,13]$. Besides, gentamicin (Gen) is known as an aminoglycoside drug that binds to the $30 \mathrm{~S}$ subunit of the ribosome to block bacterial protein synthesis [14]. Over the last several years, the effects of Amx or Gen on the human intestinal microbiota have been extensively studied [15-19]. However, very few research papers have reported their combination effects on intestinal microbial ecosystem. The simulator of the human intestinal microbial ecosystem (SHIME) model is known to be a useful tool for in vitro studies as (i) interactions between the microbiota; and (ii) the effects of prebiotics and other compounds on the microbial communities and metabolic activities [20]. To the best of our knowledge, there are only a few researches that added the antibiotics into this in vitro model, which mainly focused on the benefit of the mucosal environment, high-fiber diets, probiotic, and propionate-producing consortium in human intestinal microbiota [21-24].

At present, prebiotics, probiotics and synbiotics are increasingly used to adjust the balance of intestinal flora and keep human healthy, because they can maintain the stable abundances of probiotics such as Bifidobacteria and Lactobacilli as well as prevent the colonization of pathogenic bacteria $[25,26]$. Bifidobacterium longum is a common recognized probiotic, which has many profound benefits to human health. Bifidobacterium longum can be used to treat and prevent various forms of gastrointestinal diseases. It reported that Bifidobacterium longum may relieve the changes in the intestinal flora and gastrointestinal discomfort caused by antibiotics treatment [27]. Inulin has achieved public safety and health food certification in the United States, which was usually extracted from chicory root. Inulin would increase in the abundance of Bifidobacteria to maintain the balance of intestinal microbes [25, 28-30]. The concept of synbiotics was first proposed ten years ago, that is, the combination of prebiotics and probiotics, aiming at enhancing the effects of probiotics applied alone [31]. It suggested that synbiotics would improve the survival of probiotics after dietary supplements entering the intestine, and selectively promote the growth of beneficial bacteria and activate their metabolism [32].

Therefore, this study evaluated the in vitro ability of combined effects of Amx and Gen, and the restoration effects of probiotics or synbiotics on ARGs as well as human disease-related pathways in the simulated human gut. In this study, the composition of human intestinal microbiota was analyzed by $16 \mathrm{~S}$ rRNA gene high-throughput sequencing, the human disease-related pathways were predicted by functional predictions and the ARGs were quantified by high-throughput quantitative PCR (HT-qPCR). This study achieved a systematic investigation and precise understanding of the direct effects of combination antibiotics on the intestinal microbiota and the restoration by probiotics or synbiotics, which may be valuable for directing future work.

\section{Results}

\section{ARGs}


The relative abundance of ARGs in samples from the SHIME model were assessed at seven different time points (Fig. 1), which included samples collected after stabilization of SHIME setup for two weeks and before administration of antibiotics (Control), samples collected after the administration of a gradient of combined antibiotics with each dose for one week (AmxGen), samples collected after the after the discontinuance of antibiotics for two weeks, administration of $B$. longum for two weeks, and administration of $B$. longum and inulin for two weeks (Recovery).

As shown in Fig. 2, a total of 52 targets ARGs were detected from different group samples using a highthroughput-qPCR (HT-qPCR) technique. The heatmap showed that the relative abundances of ARGs such as aminoglycoside, beta-lactam, and multidrug resistance genes were noticeably higher in the antibiotics exposure group as compared to the control group, while the tetracycline resistance genes were lower than control. For instance, the relative log abundance of ant2ia (aminoglycoside), b/2b_tem 1 (beta-lactam), and qacedelta 1 (multidrug) were 1.8, 1.6 and $1.7 \mathrm{log}$ units higher after combined antibiotics treatment (AmxGen_1000A) than in control (Control_A2). However, tetb and tetw (tetracycline) were 1.3 and $1.7 \mathrm{log}$ units lower than the control group. After two weeks of natural recovery, probiotics or synbiotics treatment, the relative abundances of these ARGs genes were restored, and probiotics $B$. longum provided a better recovery effect. For example, the log abundances of ant2ia (aminoglycoside), b/2b_tem 1 (beta-lactam), and qacedelta 1 (multidrug) were just 0.3-0.5 log units higher in BifidobacteriumR_A than Control_A2, and tetb and tetw (tetracycline) were just 0.3-0.4 log units lower than control group.

\section{Human disease-related pathways}

The metagenomics study of the 16S rRNA gene sequence by PICRUSt revealed the gene numbers of human disease-related functional pathways in the bacterial communities of the three groups, and the genes were presented in the heatmap (Fig. 3). The heatmap showed that the gene numbers of human disease-related pathways, including cancers, drug resistance, endocrine and metabolic diseases, infectious diseases, and neurodegenerative diseases were more abundant in high dose antibiotics treatment samples than that in control group. For instance, the gene numbers of bladder cancer, cationic antimicrobial peptide resistance, insulin resistance, pertussis, and amyotrophic lateral sclerosis in the AmxGen_1000A sample were 1.5-1.7 times as that of the Control_A2 sample. After two weeks of natural recovery, probiotics or synbiotics treatment, the numbers of these genes were decreased. Natural recovery could provide a good recovery effect (these genes in AmxGen_1000A were 1.5-1.7 times as that of NatureR_A), while probiotics $B$. longum caused these genes much lower (these genes in AmxGen_1000A were 2.2-2.4 times as that of BifidobacteriumR_A).

\section{Microbiota community composition}

In this study, the effects of antibiotics treatment on gut microbial communities' composition were also investigated. Based on the 16S rRNA gene sequence analysis, the most abundant taxonomic groups assigned at the phylum level were Proteobacteria, Bacteroidetes, Firmicutes, and Fusobacteria, which account for $86.4-99.9 \%$ of the total gut microbiota (Fig. 4a). After combined amoxicillin and gentamicin 
exposure treatment with low dose, the abundances of Bacteroides (from 15.7-25.1\% to 16.6-27.3\%) and Fusobacterium (from 1.8-5.3\% to 11.5-18.7\%) increased, while the abundances of Proteobacteria (from $60.8-68.1 \%$ to $45.2-63.6 \%$ ) and Firmicutes (from 3.5-6.1\% to $0.6-2.3 \%$ ) decreased. However, after high dose antibiotics treatment, the abundance of Proteobacteria increased significantly (from 65.5-68.198.5\%), while the abundances of Bacteroides (from 22.0-25.1-1.1\%), Firmicutes (from 4.9-6.1-0.2\%) and Fusobacteria (from 1.8-0.1\%) decreased, which was more obvious in sample from ascending colon. Compared with natural recovery, prebiotics or synbiotics treatment provided a better recovery effect. Obvious decrease in the abundance of Proteobacteria (from 69.5-82.4\% to 55.0-59.1\%), and increase in abundances of Bacteroidetes (from 8.9-17.9\% to 20.2-26.9\%) and Firmicutes (from 1.9-5.6\% to 4.3$21.9 \%$ ) were seen after prebiotics or synbiotics treatment.

The abundance of bacteria at the genus level also showed distinct changes (Fig. 4b). After exposure to low concentration of amoxicillin and gentamicin, the abundance of Klebsiella reduced (from 21.6-53.1\% to $16.0-24.5 \%)$. However, after high dose antibiotics treatment, the abundance of Klebsiella significantly increased (from 44.8-53.1-73.7\%), while the abundance of Bacteroides decreased (from 18.2-20.8$1.0 \%$ ), which was also more obvious in sample from ascending colon. After two weeks of natural recovery, probiotics or synbiotics treatment, the abundances of these bacteria were recovered. The abundance of Klebsiella decreased (from 73.7\% to 40.6-42.8\%), while the abundance of Bacteroides increased (from $1.0 \%$ to $14.9-20.2 \%$ ).

The linear discriminant analysis effect size (LEfSe) comparison analysis between the three groups is shown in Fig. S1. LEfSe analysis indicated that antibiotics exposure resulted in a significant decrease in the abundance of Cloacibacillus (LDA = 4.04), accompanied by a significant increase in Escherichia/Shigella (LDA =3.99). After two weeks of natural recovery, probiotics or synbiotics treatment, the abundance of several genera increased significantly, including Anaeroglobus (LDA = 4.20), Phascolarctobacterium (LDA = 4.23), and Selenomonas (LDA = 4.60).

\section{Microbiota diversity}

Meanwhile, the fecal microbiota of alpha diversity was assessed. The taxon richness (Chao1 index), evenness (Simpson index), and diversity (Shannon index) are shown in Fig. S2. Compared with the control group, combined antibiotics exposure caused no significant difference in the microbial richness (Chao1, $P=0.913$, T test), evenness (Simpson, $P=0.859, \mathrm{~T}$ test), and diversity (Shannon, $P=0.667, \mathrm{~T}$ test). Besides, alpha diversity of the recovery microbial community still showed no difference from the control (Chao1, $P=0.731$, T test; Simpson, $P=0.955$, T test; Shannon, $P=0.937$, T test). However, the beta diversity of the microbiota communities was affected by antibiotics treatment. As shown in Fig. S3, the beta diversity results suggested that all the samples collected after combined antibiotics exposure differed from the control group, and sample AmxGen_1000A is much far away from the control group. Fig. S3 also showed that the differences still exist after two weeks of natural recovery, probiotics or synbiotics treatment. It can be seen that the ascending colon sample recovered better under natural condition, while the descending colon sample recovered better after probiotics treatment as these samples were closer to the control group. 


\section{Correlations between microbial taxa and ARGs or human disease-related pathways}

The network analysis of co-occurrence patterns between the microbial taxa and the ARG subtypes is shown in Fig. 5. It was seen that Escherichia/Shigella (significantly enriched bacteria after antibiotics treatment) was positively associated with beta-lactam and multidrug resistance genes. For example, the correlation coefficients of Escherichia/Shigella with b/1_ec, baca, and tolc were about $0.98(P<0.05)$. Cloacibacillus, the significantly decreased bacterial genus in antibiotics treatment group, was also positively associated with several ARGs. For example, the strong correlations were found in Klebsiella with $y i d y / m d t l$, teta, and aac3iia $(r=0.8-0.85, P<0.01)$.

Figure 6 shows the results of co-occurrence patterns between the microbial taxa and human diseaserelated pathways. A very similar pattern of results was observed that significantly increased bacteria Klebsiella and Escherichia/Shigella after antibiotics treatment were positively associated with most of those human disease-related pathways. Specifically, the correlation coefficients of Klebsiella with cationic antimicrobial peptide resistance, pertussis, and Salmonella infection were about $0.92(P<0.001)$ and that of Escherichia/Shigella with colorectal cancer, viral myocarditis and toxoplasmosis were $0.89(P<0.001)$.

\section{Discussion}

\section{The restoration effects of probiotics and synbiotics on microbiota community composition}

The SHIME model was stably operated in this study because the predominant phyla of Proteobacteria, Bacteriodetes, and Firmicutes in the gut microbiome was previously demonstrated by Yu's group [33]. Firmicutes and Bacteroidetes are usually dominate in the microbiota of a healthy subject. However, Proteobacteria is majoritarian in the control samples in this study. For in vivo studies, highest percentage of Proteobacteria had also been observed in fecal samples from healthy human and animals [34, 35]. Our previous in vitro studies also discovered this phenomenon [36, 37]. At the genus level, it has been verified in our previous study that after Amx exposure, Bacteroides and Klebsiella were enriched in the intestinal flora, while the abundance of Bifidobacterium was reduced [37]. These data related to Amx effects can also be supported by early reports [22, 38, 39]. Greenwood and associates investigated the effects of Gen on the gut microbiota of premature infants and indicated that this kind of antibiotic caused a significant increase in the abundance of Enterobacter[40]. Moreover, some studies have shown that the exposure to Gen would cause the significant reduction of Bacteroides and Eubacterium [41, 42]. In this study, after the combined exposure treatment of Amx and Gen, the abundance of Cloacibacillus was significantly reduced, accompanied by a significant increase in the abundance of Escherichia/Shigella. Furthermore, the abundance of Klebsiella was significantly increased while the abundance of Bacteroides was significantly reduced after high dose antibiotics exposure, and the changes in the ascending colon sample were particularly obvious. A similar study has shown that after exposure to combined 
penicillin/ampicillin and gentamicin, the abundances of Enterococcus, Staphylococcus and Klebsiella were enriched in gut flora of neonatus [43].

This study also found that, compared with natural recovery, probiotics and synbiotics treatment would provide a better recovery effects on the abundance of intestinal flora at the phylum level. At the genus level, the abundance of intestinal flora has also recovered to some extent after two weeks of natural recovery, probiotics or synbiotics treatment. For instance, the abundance of Klebsiella and Pseudomonas has declined, while the abundance of Bacteroides has increased. Our previous study showed that the abundance of Klebsiella in the intestinal flora indeed reduced in natural recovery condition, while compared with initial control group, the decrease of Bifidobacterium and the increase of Klebsiella and Bacteroides caused by Amx exposure were still obvious [37]. Our previous research also compared the recovery effects of natural condition with fecal bacteria transplantation, which indicated that the changes in intestinal flora caused by vancomycin exposure did not recover to the initial state while the recovery effects of fecal bacteria transplantation were obvious [36]. The above studies suggested that prebiotics, probiotics and fecal bacteria were effective in restoring the composition of the intestinal flora affected by antibiotics exposure. Prebiotics and probiotics can be used to keep the balance of the intestinal flora and make human healthy, with which the abundance of probiotics such as Bifidobacteria and Lactobacilli in the intestinal flora were improved and the colonization of pathogenic bacteria would be prevented [25, 26]. It reported that Bifidobacterium longum, a kind of common probiotic, may relieve the changes in the intestinal flora and gastrointestinal discomfort caused by antibiotics treatment [27]. Inulin, a kind of common prebiotic, would increase in the abundance of Bifidobacteria to maintain the balance of intestinal microbes $[25,28-30]$. Synbiotics are the combination of prebiotics and probiotics, which were designed to enhance the effects of probiotics applied alone [31]. It suggested that synbiotics would improve the survival of probiotics after dietary supplements entering the intestine, and selectively promote the growth of beneficial bacteria and activate their metabolism [32].

\section{The restoration effects of probiotics and synbiotics on microbiota diversity}

The results of the alpha diversity of the microbial community in this study showed that microbial richness, evenness, and diversity of the combined antibiotics exposure or the recovery microbial community showed no difference from the control. However, the beta diversity results indicated their differences, and the ascending colon sample recovered better under natural condition while the descending colon sample recovered better after probiotics treatment. In our previous research, the evenness and diversity of the intestinal flora were improved after Amx treatment, which were still obvious after two weeks' antibiotic discontinuance [37]. The beta diversity was also affected by Amx exposure, and the difference existed after two weeks' recovery. However, both of our unpublished data and another study's results confirmed that Gen reduced the diversity of intestinal microflora [40]. Our unpublished data also revealed that inulin could restore the alpha diversity and beta diversity of the intestinal flora well. Our previous research also carried out a comparison of the restoration effects between natural condition with fecal bacteria transplantation [36]. In that study, after vancomycin exposure or restoration, the richness, 
evenness and diversity of microbial communities were indistinguishable from control, which was similar as this study. The beta diversity results showed that the recovery effects of fecal bacteria transplantation were much more obvious than natural condition. The above studies generally suggested that prebiotics, probiotics and fecal bacteria transplantation are more effective in restoring the diversity of intestinal flora.

Some studies have shown that administration of antibiotics is significantly associated with decrease in microbial community diversity and richness [44]. The results of this study indicated that combined antibiotics exposure did not have a significant impact on microbiome diversity, which is consistent with the conclusions as our previous vancomycin exposure study [36]. Some studies have also found that exposure to beta-lactam antibiotics does not have a significant effect on microbiome diversity [17-19]. Our previous research even confirmed that Amx exposure increased microbial diversity [37]. As discussed earlier, prebiotics and probiotics can be used to keep the balance of the intestinal flora and make human healthy, so they could also have a certain recovery effect on the diversity of intestinal flora. The diversity and balance of the intestinal flora has been considered to be closely related to many aspects of human health, including immunity, metabolism and neurological function [45]. Studies have shown that the consumption of Bifidobacterium longum would help recover the changed diversity of the intestinal flora caused by antibiotics and restore intestinal flora balance [27, 46, 47]. Inulin could also restore the affected alpha diversity and beta diversity of intestinal microbes. Most studies have shown that the alpha diversity of intestinal flora would decrease or have no evident changes after supplementation of inulin, and these studies also suggested that it would cause a significant change in beta diversity afterwards $[28-30,48]$. These studies are consistent with the results of this study.

\section{The restoration effects of probiotics and synbiotics on functional genes}

This study discovered that the relative abundance of most ARGs and the gene numbers of human disease-related pathways increased after the combined antibiotics exposure. After two weeks of natural recovery, probiotics or synbiotics treatment, the number of these genes decreased. Among these recovery methods, probiotics provided a better recovery effect by dropping more genes. Antibiotics may also cause the destruction of the microbiota and immune homeostasis and lead to disease while promoting the clearance of targeted infections [49]. Amx alone may selectively promote the increase of human diseaserelated genes and activate innate immunity, causing infectious diseases such as urinary tract infection, diarrhea and liver abscess [50-53]. Some other studies have shown that Amx would enrich the betalactam resistance genes and corresponding antibiotic resistant bacteria in the intestine [39, 54]. Taking Gen would also promote the spread of antibiotic resistant bacteria and ARGs $[15,16]$. The above conclusions would help us infer the combined exposure effects on the increase of ARGs and human disease-related genes in this study.

This study also found that these changed genes were positively correlated with the bacterial genera that changed significantly after combined antibiotic exposure, which was similar to our previous studies [36, 
37]. Therefore, the changes of these genes mainly attributed to the bacterial shift caused by combined antibiotics exposure. The previous section has explored the restoration effects of prebiotics and probiotics on the microbiota community composition, which therefore help explain their restoration effects on genetic level. The proliferation of Klebsiella in the intestines may cause different kinds of intestinal infections $[55,56]$. Some studies have shown that pneumonia, Crohn's disease, colitis, cystitis, wound infection and liver abscess are all related to the proliferation of Klebsiella in the human intestine $[57,58]$. There are also some studies have confirmed that Escherichia/Shigella is related to human diseases such as enteritis, metabolic diseases, cancer, and cardiovascular diseases [59-62]. In addition, some other studies have shown that Escherichia/Shigella is the host of tetracycline and multidrug resistance genes, making this strain has the characteristic of natural resistance to these kinds of antibiotics $[63,64]$. These research conclusions all give the explanations to the phenomena found in this study.

\section{Perspectives}

The findings in this study suggested several numbers of opportunities for additional study. First, expansion of the analysis to incorporate multiple-omics approaches of the metagenome, metatranscriptome, and metabolome would help us confirm the genome composition and expression of the gut microbiota and understand how antibiotic affected human disease and drug resistance. It is also of interest to inoculate the microbiota mixture from several patients to discover the different effects of antibiotic between healthy and sick individuals. Further in vivo studies aimed to verify whether the findings in this in vitro study reflect the reality would also be interesting, which will provide new insights to measure how antibiotic affects the gut microbiota and the associated disease.

\section{Conclusion}

In this study, a combination of Amx and Gen was confirmed to promote the increase of most ARGs and the disease-related pathways, which may be better restored by probiotics treatment. The results of the alpha diversity of the combined antibiotics exposure or the recovery microbial community showed no difference from the control. However, the beta diversity results indicated their differences, and the ascending colon sample recovered better under natural condition while the descending colon sample recovered better after probiotics treatment. Combination effects on the genetic level might attribute to microbiota shift, which were explained well by the phenomenon that Escherichia/Shigella was positively associated with the ARGs, and Klebsiella and Escherichia/Shigella were positively related to the human disease-related pathways. These results might be valuable to direct the future work and opened up new perspectives to address the direct effects of combine antibiotics on the intestinal microbiota and find a promising strategy to restore the antibiotics associated dysbiosis of gut microbiota.

\section{Methods}

\section{Antibiotics treatment and samples collection}


In this study, the SHIME was constructed using four double-jacketed reactors designated as the stomach, small intestine, ascending colon, and descending colon, respectively (Fig. 1). The last two reactors were inoculated with a mixture of fecal microbiota from one healthy adult volunteer, who did not suffer by any gastrointestinal disease or take antibiotics in the past six months, on account of the differences between individuals may be alleviated by same culture condition [65]. The details of the SHIME system and the startup process are summarized in the Supplementary material.

During the first three weeks of the experiment, nutritional medium was added to the reactors to stabilize the microbial community. After this period, a gradient of combined antibiotics (nutritional medium +100 $\mathrm{mg} \cdot \mathrm{L}^{-1} \mathrm{Amx}+50 \mathrm{mg} \cdot \mathrm{L}^{-1} \mathrm{Gen}$ for one week, then nutritional medium $+1000 \mathrm{mg} \cdot \mathrm{L}^{-1} \mathrm{Amx}+500 \mathrm{mg} \cdot \mathrm{L}^{-1}$ Gen for one week) were added. Then, the SHIME was sequentially exposed to nutritional medium $+10^{9}$ $\mathrm{CFU} \cdot \mathrm{mL}^{-1}$ B. longum for two weeks, nutritional medium $+10^{9} \mathrm{CFU} \cdot \mathrm{mL}^{-1}$ B. longum for two weeks, and nutritional medium $+10^{9} \mathrm{CFU} \cdot \mathrm{mL}^{-1}$ B. longum $+4000 \mathrm{mg} \cdot \mathrm{L}^{-1}$ inulin for two weeks. The details of the SHIME system and the startup process are summarized in the Supplementary material.

Liquid samples (mixtures of fecal microbiota with SHIME feed) were collected from simulated ascending colon and descending colon vessels at seven time points, as detailed in Fig. 1. Based on the situation, these samples can be classified into three groups. Specifically, the first group of samples were collected after stabilization of SHIME setup for two weeks (Control_A1 and Control_D1 from ascending colon and descending colon, respectively) and before administration of antibiotics (Control_A2 and Control_D2). The second group sampled after the administration of low dose antibiotics for one week (AmxGen_100A and AmxGen_100D) and high dose antibiotics one week (AmxGen_1000A and AmxGen_1000D). Finally, the third group of samples were collected after the discontinuance of antibiotics for two weeks (NatureR_A and NatureR_D), administration of B. longum for two weeks (BifidobacteriumR_A and BifidobacteriumR_D), and administration of $B$. longum and inulin for two weeks (BifidolnulinR_A and BifidolnulinR_D). Each sample is a mixture of three samples collected at specific time intervals in a day. The samples were stored at $-80^{\circ} \mathrm{C}$ for further analyses.

\section{S rRNA gene sequencing and analysis}

Total DNA was extracted from the samples using the E.Z.N.A. stool DNA Kit (Omega, USA) according to the manufacturer's protocols. The V3-V4 region of the bacterial 16S rRNA gene was amplified by polymerase chain reaction (PCR). The raw reads of the sequences were deposited into the NCBI Sequence Read Archive (SRA) database under the accession number SRR11342782-11342795. The raw Illumina fastq files were de-multiplexed, quality-filtered, and analyzed using Quantitative Insights Into Microbial Ecology (QIIME) [66]. The 16S rRNA gene sequences were further taxonomically classified using the Ribosomal Database Project (RDP) classifier 2.0.1 [67].

The effects of antibiotics on alpha diversity, the taxon richness (Chao1 index), evenness (Simpson index), and diversity (Shannon index) were calculated for all the samples as previous did [36, 68]. In addition, beta diversity of the microbiota communities at baseline and after antibiotics were portrayed by nonmetric multidimensional scaling (NMDS) and principal coordinate analysis (PCoA) of weighted and 
unweighted UniFrac distances [69]. Linear discriminant analysis effect size (LEfSe) was performed to determine bacterial taxa that were significantly differed between the six groups using the Galaxy application tool [70]. Functional predictions of microbial community were performed to visualize the distribution of human disease-related pathways in the six groups using Phylogenetic Investigation of Communities by Reconstruction of Unobserved States (PICRUSt) [71]. The accuracy of PICRUSt for the detection of more challenging functional groups was good (min. accuracy $=0.82$ ), suggesting that their inference of gene abundance across various types of functions was reliable, and PICRUSt predictions had high agreement with metagenome sample abundances across all body sites (Spearman $r=0.82, P<$ 0.001). These analyses were conducted by BioMarker Technology Co., Ltd (Beijing, China). The details of $16 \mathrm{~s}$ rRNA gene amplification and sequencing, taxonomical classification, LEfSe analysis, and functional predictions are described in the Supplementary material.

\section{High-throughput quantitative PCR (HT-qPCR) and analysis}

High-throughput-qPCR reactions were performed using the Wafergen SmartChip Real-time PCR system as previous did $[36,68]$. The reactions were conducted by Anhui MicroAnaly Gene Technologies Co., Ltd (Anhui, China). A total of 108 primer sets were chosen (Excel S1), which included 102 primer sets to target the almost all major classes of antibiotic resistance genes (ARGs) found in the microbiota of Chinese human gut [72], along with five mobile genetic elements (MGEs) and one 16S rRNA gene. The results were analyzed using the SmartChip qPCR Software. Data with multiple melting peaks or amplification beyond the range ( 0 ) were excluded and then screened with conditions that a threshold cycle (CT) must be $<31$, and positive samples should have three replicates simultaneously. The details of HT-qPCR analysis are described in the Supplementary material.

\section{Data analysis}

All the results were expressed as mean values and standard deviations. The statistical analysis was performed with SPSS 17.0 software (SPSS Inc., Chicago, III., U.S.A.). The T-test was conducted to compare the differences between the groups, and all the statistical tests were two-tailed. The statistical significance was set at three different levels ( ${ }^{\star} P<0.05,{ }^{*} P<0.01$, and $\left.{ }^{\star} * \star P<0.001\right)$. Correlations between the microbiota and human disease-related pathways or ARGs were analyzed using the Spearman test in $\mathrm{R}$ with the 'vegan' package. The correlations between the pairs of variables were considered to be significant at $r>0.6$, and $P$ values were $<0.05$. The Gephi $(V 0.9 .1)$ software was used to visualize the bipartite network graphs using the Force Atlas algorithm.

\section{Declarations}

\section{Availability of data and materials}

The datasets and scripts developed and generated in this manuscript are included within the manuscript and its supporting files. 


\section{Consent for publication}

Not applicable.

\section{Ethics approval and consent to participate}

The study was approved by the Biomedical Ethics Committees of Nankai University. The participant has given written, informed consent to understand the study's purpose, procedures, risks, benefits, and rights.

\section{Competing interest}

The authors declare that they have no competing interests.

\section{Funding}

This work was supported by a grant from the project of 'Jiangsu Specially-Appointed Professor' (1421071901002).

\section{Authors' contributions}

Lei Liu: Methodology, Investigation, Formal analysis, Writing - original draft, Software. Hongmei Qi: Investigation. Pengcheng Suo: Investigation. Huai Lin: Investigation. Siyi Wang: Investigation. Xiaojun Zuo: Funding acquisition, Writing - review \& editing.

\section{Acknowledgments}

The authors express the sincerest thanks to Professor Bing Wu (School of the Environment in Nanjing University, Nanjing in China) for the guidance of SHIME, and Guangzhou Gene Denovo Co., Ltd, and Anhui MicroAnaly Gene Technologies Co., Ltd for genome sequencing and analysis.

\section{References}

1. Ashbolt NJ, Amezquita A, Backhaus T, Borriello P, Brandt KK, Collignon P, et al. Human Health Risk Assessment (HHRA) for Environmental Development and Transfer of Antibiotic Resistance. Environ Health Perspect. 2013;121(9):993-1001.

2. Ben YJ, Fu CX, Hu M, Liu L, Wong MH, Zheng CM. Human health risk assessment of antibiotic resistance associated with antibiotic residues in the environment: A review. Environ Res. 2019;169:483-93. 
3. Le Page G, Gunnarsson L, Snape J, Tyler CR. Integrating human and environmental health in antibiotic risk assessment: A critical analysis of protection goals, species sensitivity and antimicrobial resistance. Environ Int. 2017;109:155-69.

4. Nnadozie $\mathrm{CE}$, Odume ON. Freshwater environments as reservoirs of antibiotic resistant bacteria and their role in the dissemination of antibiotic resistance genes. Environ Pollut. 2019;254.

5. Khan I, Yasir M, Azhar El, Kumosani T, Barbour EK, Bibi F, et al. Implication of gut microbiota in human health. CNS Neurol Disord Drug Targets. 2014;13(8):1325-33.

6. Wang Q, Duan YJ, Wang SP, Wang LT, Hou ZL, Cui YX, et al. Occurrence and distribution of clinical and veterinary antibiotics in the faeces of a Chinese population. J Hazard Mater. 2020;383:121129.

7. Cabreiro F, Au C, Leung KY, Vergara-Irigaray N, Cocheme HM, Noori T, et al. Metformin retards aging in C. elegans by altering microbial folate and methionine metabolism. Cell. 2013;153(1):228-39.

8. Qin JJ, Li RQ, Raes J, Arumugam M, Burgdorf KS, Manichanh C, et al. A human gut microbial gene catalogue established by metagenomic sequencing. Nature. 2010;464(7285):59-65.

9. Bengtsson-Palme J, Kristiansson E, Larsson DGJ. Environmental factors influencing the development and spread of antibiotic resistance. FEMS Microbiol Rev. 2018;42(1):68-80.

10. Stecher B, Maier L, Hardt WD. 'Blooming' in the gut: how dysbiosis might contribute to pathogen evolution. Nat Rev Microbiol. 2013;11(4):277-84.

11. Barraud O, Peyre M, Couve-Deacon E, Chainier D, Bahans C, Guigonis V, et al. Antibiotic resistance acquisition in the first week of life. Front Microbiol. 2018;9:1467.

12. Zapata HJ, Quagliarello VJ. The microbiota and microbiome in aging: Potential implications in health and age-related diseases. J Am Geriatr Soc. 2015;63(4):776-81.

13. Zhang Q, Ying G, Pan C, Liu Y, Zhao J. Comprehensive evaluation of antibiotics emission and fate in the river basins of China: source analysis, multimedia modeling, and linkage to bacterial resistance. Environ Sci Technol. 2015;49(11):6772-82.

14. Puia-Dumitrescu M, Bretzius OM, Brown N, Fitz-Henley JA, Ssengonzi R, Wechsler CS, et al. Evaluation of Gentamicin Exposure in the Neonatal Intensive Care Unit and Hearing Function at Discharge. The Journal of pediatrics. 2018.

15. Barros JCD, Pinheiro SR, Bozza M, Gueiros FJ, Bello AR, Lopes UG, et al. Evidences of gentamicin resistance amplification in Klebsiella pneumoniae isolated from faeces of hospitalized newborns. Mem Inst Oswaldo Cruz. 1999;94(6):795-802.

16. Fouhy F, Guinane CM, Hussey S, Wall R, Ryan CA, Dempsey EM, et al. High-Throughput Sequencing Reveals the Incomplete, Short-Term Recovery of Infant Gut Microbiota following Parenteral Antibiotic Treatment with Ampicillin and Gentamicin. Antimicrob Agents Chemother. 2012;56(11):5811-20.

17. Pallav K, Dowd SE, Villafuerte J, Yang X, Kabbani T, Hansen J, et al. Effects of polysaccharopeptide from Trametes versicolor and amoxicillin on the gut microbiome of healthy volunteers: a randomized clinical trial. Gut Microbes. 2014;5(4):458-67. 
18. Reijnders D, Goossens GH, Hermes GD, Neis EP, van der Beek CM, Most J, et al. Effects of gut microbiota manipulation by antibiotics on host metabolism in obese humans: a randomized doubleblind placebo-controlled trial. Cell Metab. 2016;24(1):63-74.

19. Zaura E, Brandt BW, Teixeira de Mattos MJ, Buijs MJ, Caspers MP, Rashid MU, et al. Same exposure but two radically different responses to antibiotics: Resilience of the salivary microbiome versus long-term microbial shifts in feces. MBio. 2015;6(6):e01693-15.

20. Marzorati M, Van de Wiele T. An advanced in vitro technology platform to study the mechanism of action of prebiotics and probiotics in the gastrointestinal tract. J Clin Gastroenterol. 2016;50:124-S5.

21. Ichim TE, Kesari S, Shafer K. Protection from chemotherapy- and antibiotic-mediated dysbiosis of the gut microbiota by a probiotic with digestive enzymes supplement. Oncotarget. 2018;9(56):3091935.

22. Marzorati M, Vilchez-Vargas R, Bussche JV, Truchado P, Jauregui R, El Hage RA, et al. High-fiber and high-protein diets shape different gut microbial communities, which ecologically behave similarly under stress conditions, as shown in a gastrointestinal simulator. Mol Nutr Food Res. 2017;61(1):1600150.

23. Van den Abbeele P, Roos S, Eeckhaut V, MacKenzie DA, Derde M, Verstraete W, et al. Incorporating a mucosal environment in a dynamic gut model results in a more representative colonization by Lactobacilli. Microb Biotechnol. 2012;5(1):106-15.

24. El Hage R, Hernandez-Sanabria E, Calatayud Arroyo M, Props R, Van de Wiele T. Propionateproducing consortium restores antibiotic-induced dysbiosis in a dynamic in vitro model of the human intestinal microbial ecosystem. Front Microbiol. 2019;10:1206.

25. Dahiya DK, Renuka, Puniya M, Shandilya UK, Dhewa T, Kumar N, et al. Gut Microbiota Modulation and Its Relationship with Obesity Using Prebiotic Fibers and Probiotics: A Review. Front Microbiol. 2017;8.

26. Vaughn BP, Rank KM, Khoruts A. Fecal Microbiota Transplantation: Current Status in Treatment of GI and Liver Disease. Clin Gastroenterol Hepatol. 2019;17(2):353-61.

27. Orrhage K, Sjostedt S, Nord CE. Effect of supplements with lactic acid bacteria and oligofructose on the intestinal microflora during administration of cefpodoxime proxetil. J Antimicrob Chemother. 2000;46(4):603-12.

28. Healey G, Murphy R, Butts C, Brough L, Whelan K, Coad J. Habitual dietary fibre intake influences gut microbiota response to an inulin-type fructan prebiotic: a randomised, double-blind, placebocontrolled, cross-over, human intervention study. Br J Nutr. 2018;119(2):176-89.

29. Reimer RA, Willis HJ, Tunnicliffe JM, Park H, Madsen KL, Soto-Vaca A. Inulin-type fructans and whey protein both modulate appetite but only fructans alter gut microbiota in adults with overweight/obesity: A randomized controlled trial. Mol Nutr Food Res. 2017;61(11).

30. Baxter NT, Schmidt AW, Venkataraman A, Kim KS, Waldron C, Schmidt TM. Dynamics of Human Gut Microbiota and Short-Chain Fatty Acids in Response to Dietary Interventions with Three Fermentable Fibers. mBio. 2019;10(1). 
31. Markowiak P, Slizewska K. Effects of Probiotics, Prebiotics, and Synbiotics on Human Health. Nutrients. 2017;9(9).

32. Pandey KR, Naik SR, Vakil BV. Probiotics, prebiotics and synbiotics- a review. J Food Sci Technol. 2015;52(12):7577-87.

33. Yu H, Wu B, Zhang X, Liu S, Yu J, Cheng S, et al. Arsenic metabolism and toxicity influenced by ferric iron in simulated gastrointestinal tract and the roles of gut microbiota. Environ Sci Technol. 2016;50(13):7189-97.

34. Gao Z, Guo B, Gao R, Zhu Q, Qin H. Microbiota disbiosis is associated with colorectal cancer. Front Microbiol. 2015;6:20.

35. Li S, Zhang C, Gu Y, Chen L, Ou S, Wang Y, et al. Lean rats gained more body weight than obese ones from a high-fibre diet. Br J Nutr. 2015;114(8):1188-94.

36. Liu L, Wang Q, Wu X, Qi H, Das R, Lin H, et al. Vancomycin exposure caused opportunistic pathogens bloom in intestinal microbiome by simulator of the human intestinal microbial ecosystem (SHIME). Environ Pollut. 2020:114399.

37. Liu L, Wang Q, Lin H, Das R, Wang S, Qi H, et al. Amoxicillin Increased Functional Pathway Genes and Beta-Lactam Resistance Genes by Pathogens Bloomed in Intestinal Microbiota Using a Simulator of the Human Intestinal Microbial Ecosystem. Front Microbiol. 2020;11:1213.

38. Ladirat SE, Schoterman MHC, Rahaoui H, Mars M, Schuren FHJ, Gruppen H, et al. Exploring the effects of galacto-oligosaccharides on the gut microbiota of healthy adults receiving amoxicillin treatment. Br J Nutr. 2014;112(4):536-46.

39. Oh B, Kim BS, Kim JW, Kim JS, Koh SJ, Kim BG, et al. The effect of probiotics on gut microbiota during the Helicobacter pylori eradication: randomized controlled trial. Helicobacter. 2016;21(3):16574.

40. Greenwood C, Morrow AL, Lagomarcino AJ, Altaye M, Taft DH, Yu Z, et al. Early Empiric Antibiotic Use in Preterm Infants Is Associated with Lower Bacterial Diversity and Higher Relative Abundance of Enterobacter. J Pediatr. 2014;165(1):23-9.

41. Xiao Y, Zhou K, Lu Y, Yan W, Cai W, Wang Y. Administration of antibiotics contributes to cholestasis in pediatric patients with intestinal failure via the alteration of FXR signaling. Exp Mol Med. 2018;50(12):1-14.

42. Johnson LP, Walton GE, Psichas A, Frost GS, Gibson GR, Barraclough TG. Prebiotics Modulate the Effects of Antibiotics on Gut Microbial Diversity and Functioning in Vitro. Nutrients. 2015;7(6):448097.

43. Kozyrskyj AL, Bridgman SL, Tun MH. The impact of birth and postnatal medical interventions on infant gut microbiota. 2017.

44. Dethlefsen L, Huse S, Sogin ML, Relman DA. The pervasive effects of an antibiotic on the human gut microbiota, as revealed by deep 16S rRNA sequencing. PLoS Biol. 2008;6(11):e280.

45. Valdes AM, Walter L, Segal E, Spector TD. Role of the gut microbiota in nutrition and health. Bmj-Brit Med J. 2018;361. 
46. Mizuta M, Endo I, Yamamoto S, Inokawa H, Kubo M, Udaka T, et al. Perioperative supplementation with bifidobacteria improves postoperative nutritional recovery, inflammatory response, and fecal microbiota in patients undergoing colorectal surgery: a prospective, randomized clinical trial. Bioscience of Microbiota, Food and Health. 2016;35(2):77-87.

47. Odamaki T, Kato K, Sugahara H, Xiao JZ, Abe F, Benno Y. Effect of probiotic yoghurt on animal-based diet-induced change in gut microbiota: an open, randomised, parallel-group study. Benef Microbes. 2016;7(4):473-84.

48. Vandeputte D, Falony G, Vieira-Silva S, Wang J, Sailer M, Theis S, et al. Prebiotic inulin-type fructans induce specific changes in the human gut microbiota. Gut. 2017;66(11):1968-74.

49. Ubeda C, Pamer EG. Antibiotics, microbiota, and immune defense. Trends Immunol. 2012;33(9):45966.

50. Morley VJ, Woods RJ, Read AF. Bystander selection for antimicrobial resistance: Implications for patient health. Trends Microbiol. 2019;27(10):864-77.

51. Oh J, Patel J, Park HB, Crawford JM. beta-Lactam biotransformations activate innate immunity. J Org Chem. 2018;83(13):7173-9.

52. Kabbani TA, Pallav K, Dowd SE, Villafuerte-Galvez J, Vanga RR, Castillo NE, et al. Prospective randomized controlled study on the effects of Saccharomyces boulardii CNCM I-745 and amoxicillinclavulanate or the combination on the gut microbiota of healthy volunteers. Gut Microbes. 2017;8(1):17-32.

53. Lin Y-T, Liu C-J, Yeh Y-C, Chen T-J, Fung C-P. Ampicillin and amoxicillin use and the risk of Klebsiella pneumoniae liver abscess in Taiwan. J Infect Dis. 2013;208(2):211-7.

54. Forssten S, Evans M, Wilson D, Ouwehand AC. Influence of a probiotic mixture on antibiotic induced microbiota disturbances. World J Gastroenterol. 2014;20(33):11878-85.

55. Gorrie C, Mirceta M, Wick R, Edwards D, Thomson N, Strugnell R, et al. Gastrointestinal carriage is a major reservoir of Klebsiella pneumoniae infection in intensive care patients. Clin Infect Dis. 2017;65(2):208-15.

56. Wiener-Well Y, Rudensky B, Yinnon AM, Kopuit P, Schlesinger Y, Broide E, et al. Carriage rate of carbapenem-resistant Klebsiella pneumoniae in hospitalised patients during a national outbreak. J Hosp Infect. 2010;74(4):344-9.

57. Atarashi K, Suda W, Luo C, Kawaguchi T, Motoo I, Narushima S, et al. Ectopic colonization of oral bacteria in the intestine drives TH1 cell induction and inflammation. Science. 2017;358(6361):35965.

58. Schneditz G, Rentner J, Roier S, Pletz J, Herzog KA, Bucker R, et al. Enterotoxicity of a nonribosomal peptide causes antibiotic-associated colitis. Proc Natl Acad Sci U S A. 2014;111(36):13181-6.

59. Thorkildsen LT, Nwosu FC, Avershina E, Ricanek P, Perminow G, Brackmann S, et al. Dominant Fecal Microbiota in Newly Diagnosed Untreated Inflammatory Bowel Disease Patients. Gastroent Res Pract. 2013. 
60. Mei QX, Huang CL, Luo SZ, Zhang XM, Zeng Y, Lu YY. Characterization of the duodenal bacterial microbiota in patients with pancreatic head cancer vs. healthy controls. Pancreatology. 2018;18(4):438-45.

61. Mushtaq N, Hussain S, Zhang SR, Yuan L, Li H, Ullah S, et al. Molecular characterization of alterations in the intestinal microbiota of patients with grade 3 hypertension. Int $\mathrm{J}$ Mol Med. 2019;44(2):513-22.

62. Tao SB, Li LZ, Li L, Liu Y, Ren Q, Shi M, et al. Understanding the gut-kidney axis among biopsy-proven diabetic nephropathy, type 2 diabetes mellitus and healthy controls: an analysis of the gut microbiota composition. Acta Diabetol. 2019;56(5):581-92.

63. Xiong WG, Wang YL, Sun YX, Ma LP, Zeng QL, Jiang XT, et al. Antibiotic-mediated changes in the fecal microbiome of broiler chickens define the incidence of antibiotic resistance genes. Microbiome. 2018;6.

64. Xiong WG, Sun YX, Ding XY, Wang MZ, Zeng ZL. Selective pressure of antibiotics on ARGs and bacterial communities in manure-polluted freshwater-sediment microcosms. Front Microbiol. 2015;6.

65. Van den Abbeele P, Belzer C, Goossens M, Kleerebezem M, De Vos WM, Thas O, et al. Butyrateproducing Clostridium cluster XIVa species specifically colonize mucins in an in vitro gut model. ISME J. 2013;7(5):949-61.

66. Caporaso JG, Kuczynski J, Stombaugh J, Bittinger K, Bushman FD, Costello EK, et al. QIIME allows analysis of high-throughput community sequencing data. Nat Methods. 2010;7(5):335-6.

67. Wang Q, Garrity GM, Tiedje JM, Cole JR. Naive Bayesian classifier for rapid assignment of rRNA sequences into the new bacterial taxonomy. Appl Environ Microbiol. 2007;73(16):5261-7.

68. Liu L, Wang Q, Lin H, Das R, Wang S, Qi H, et al. Amoxicillin Increased Functional Pathway Genes and Beta-Lactam Resistance Genes by Pathogens Bloomed in Intestinal Microbiota Using a Simulator of the Human Intestinal Microbial Ecosystem. Front Microbiol. 2020;11:1213.

69. Lozupone C, Knight R. UniFrac: a new phylogenetic method for comparing microbial communities. Appl Environ Microbiol. 2005;71(12):8228-35.

70. Segata N, Izard J, Waldron L, Gevers D, Miropolsky L, Garrett WS, et al. Metagenomic biomarker discovery and explanation. Genome Biol. 2011;12(6):R60.

71. Langille MGI, Zaneveld J, Caporaso JG, McDonald D, Knights D, Reyes JA, et al. Predictive functional profiling of microbial communities using 16S rRNA marker gene sequences. Nat Biotechnol. 2013;31(9):814-21.

72. Hu Y, Yang X, Qin J, Lu N, Cheng G, Wu N, et al. Metagenome-wide analysis of antibiotic resistance genes in a large cohort of human gut microbiota. Nat Commun. 2013;4:2151-249.

\section{Supplementary Information}

Additional file 1: Supplementary information file. Fig. S1. LDA score and cladogram of LEfSe comparison analysis among control (green), antibiotics treatment (red), and recovery (blue) groups. The green, red, or 
blue shading depicts bacterial taxa that were significantly higher in either the control, antibiotics treatment or recovery groups, as indicated. Selection of discriminative taxa between the control and gentamycin groups or between the control and inulin groups were based on an LDA score cutoff of 3.0, and differences in the relative abundances of taxa were statistically determined based on a MannWhitney test at a significance level of 0.05 . Fig. S2. Gut microbiota alpha diversity of the control (green), antibiotics treatment (red), and recovery (blue) groups ( ${ }^{\star \star \star} p<0.001,{ }^{*} p<0.01,{ }^{*} p<0.05$ ). The Chao1 index (a) was used to calculate the community richness, Simpson index (b) was used to calculate the community evenness, and Shannon index (c) was used to calculate the community diversity. Fig. S3. Gut microbiota beta diversity of the control (green), antibiotics treatment (red), and recovery (blue) groups. Unweighted (a) and weighted (b) PCoA, and unweighted (c) and weighted (d) NMDS of UniFrac distances of samples in three groups (control, gentamycin and inulin).

Additional file 2: Excel S1 Primer sets of HT-qPCR reactions using Wafergen SmartChip Real-time PCR system.

\section{Figures}

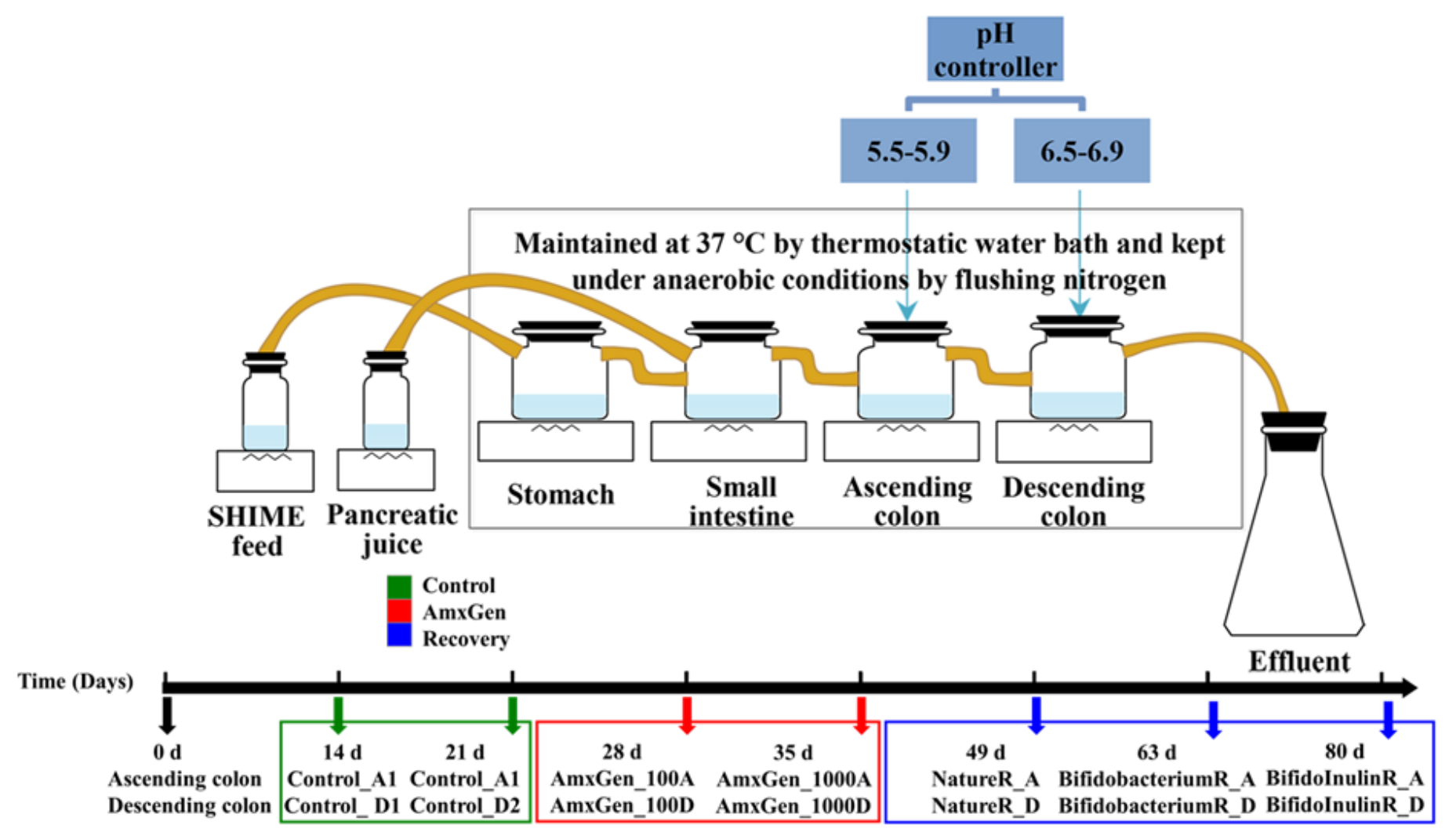

Figure 1

Schematic of designed SHIME model and sampling time points setup. 


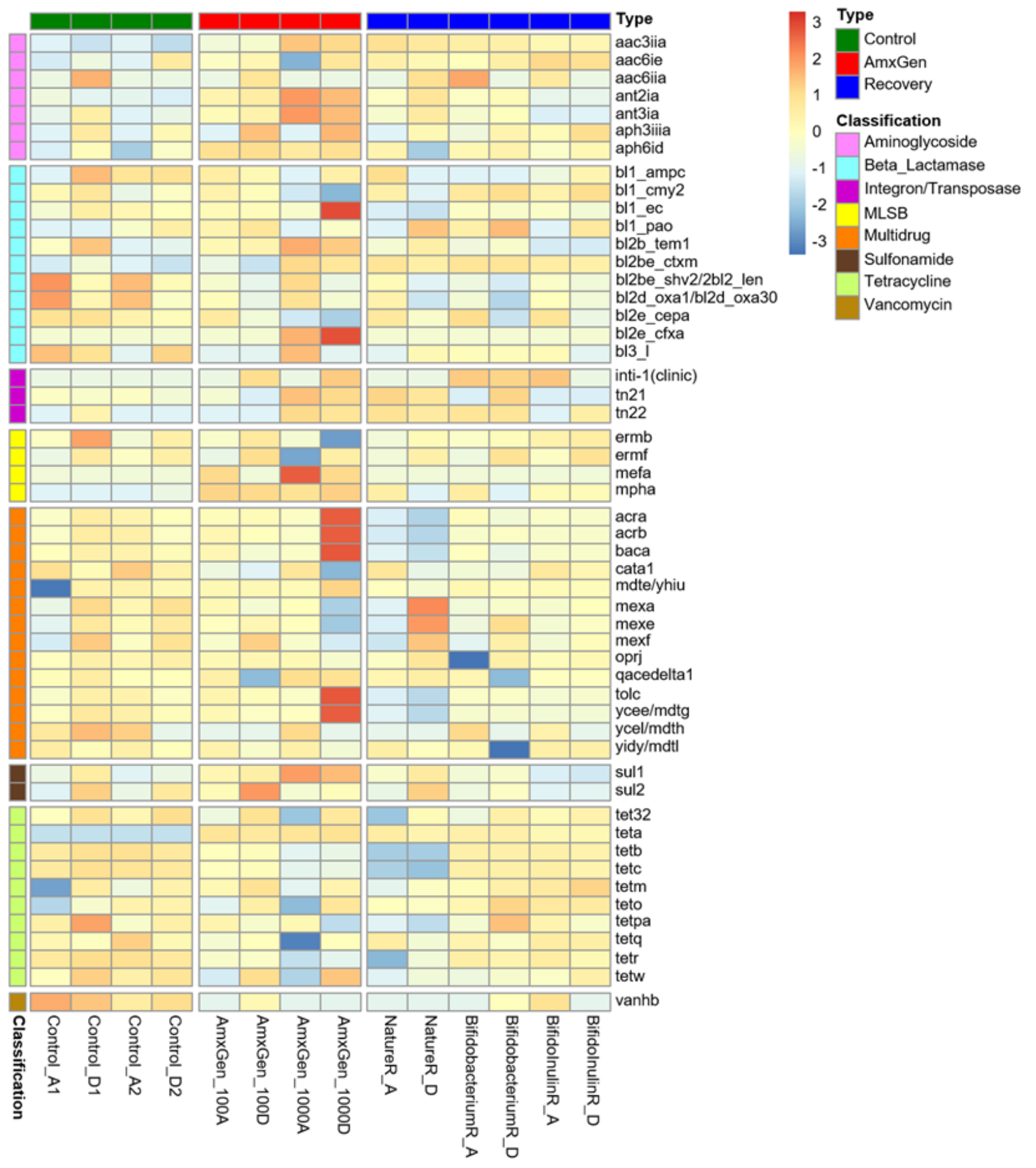

Figure 2

Heatmap of antibiotic resistance genes (ARGs) in the three groups. Heatmap colors reflect relative abundance of ARGs from low (blue) to high (red). 


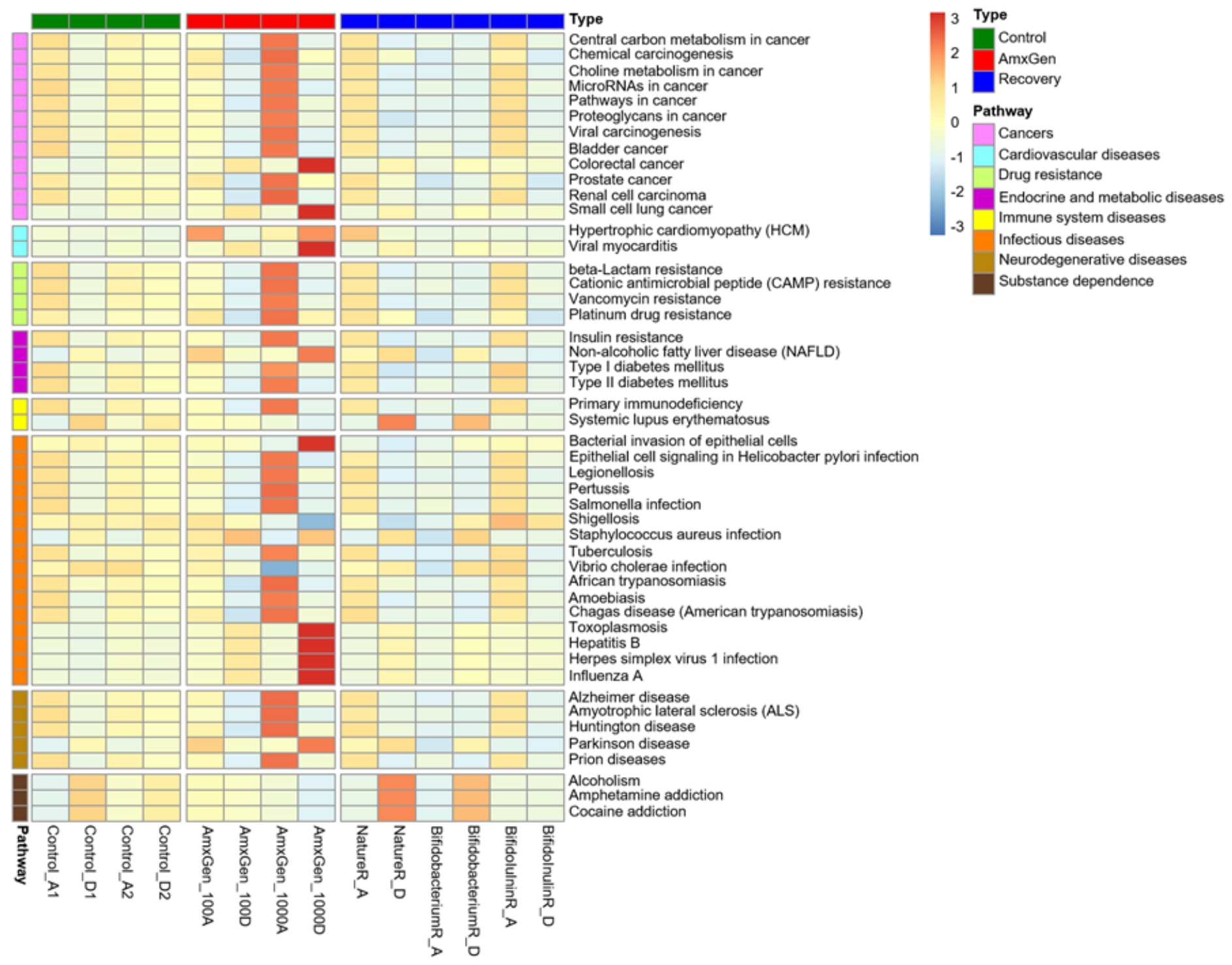

Figure 3

Heatmap of human disease-related pathways in the three groups. Heatmap colors reflect gene numbers of human disease-related pathways from low (blue) to high (red). 


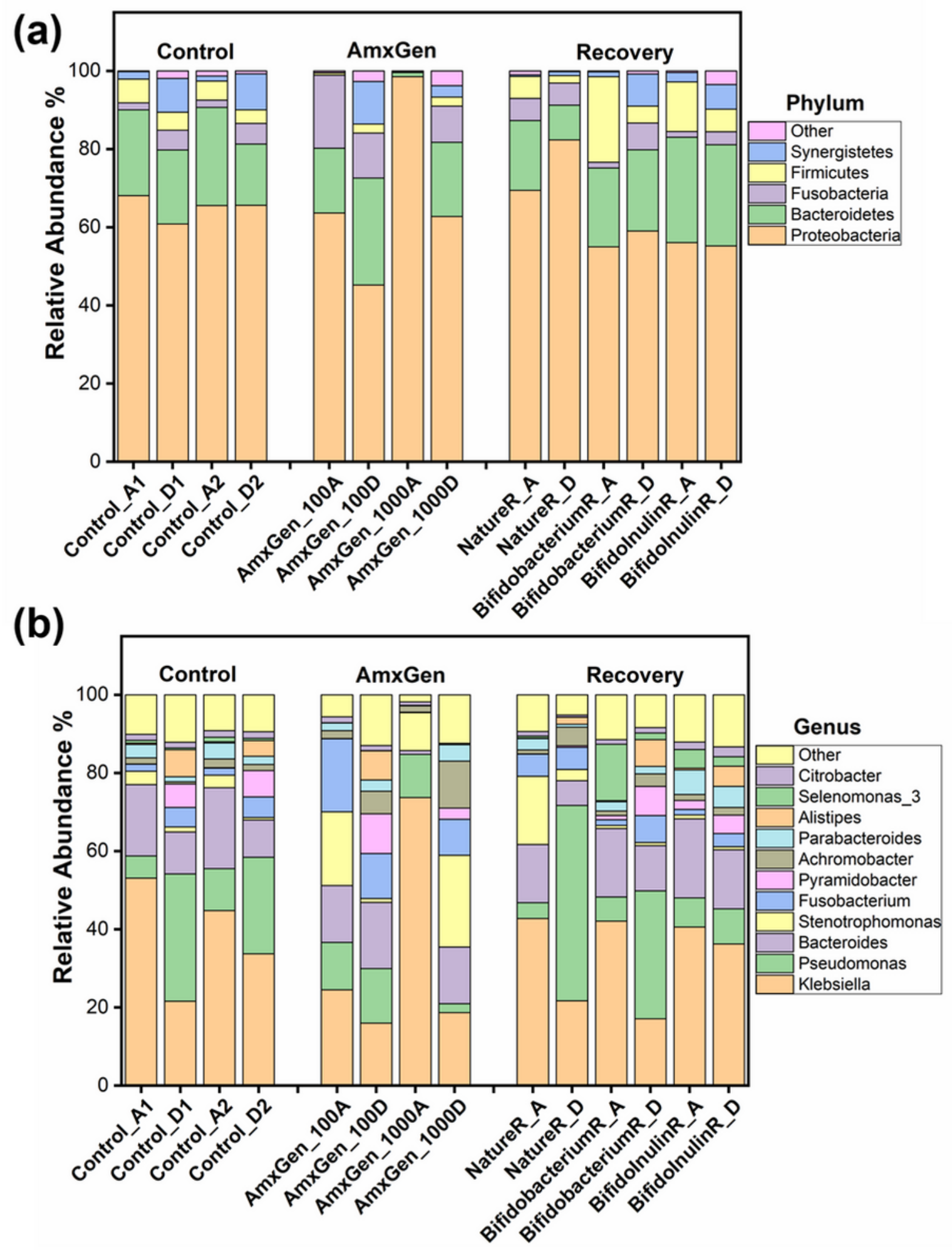

Figure 4

Composition of microbial community at phylum (a) and genus level (b). 


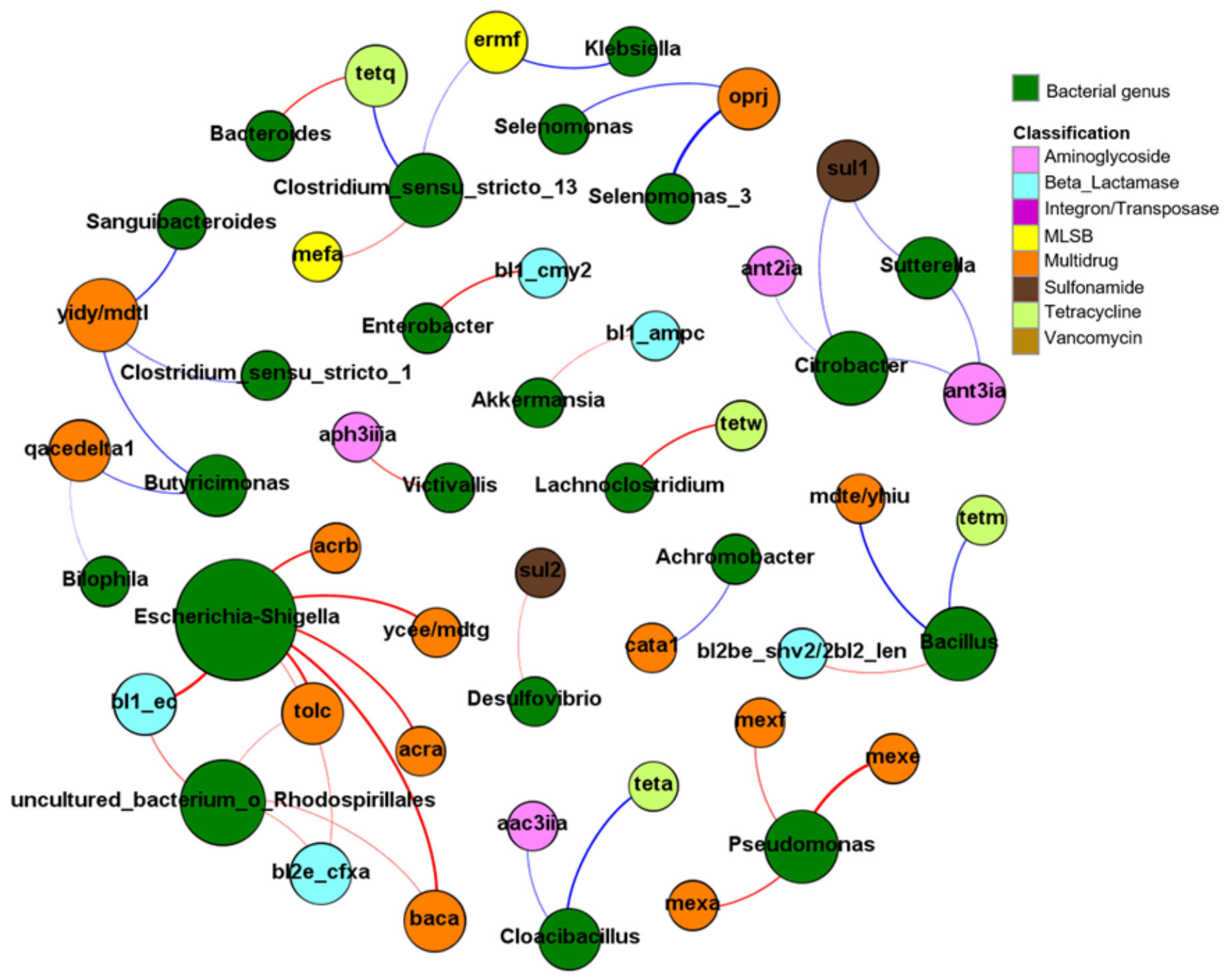

Figure 5

Network analysis revealing the co-occurrence patterns between microbial taxa and ARG subtypes. The nodes in Network were colored according to ARG types. The edges were colored according to positive (red) or negative (blue) correlation. A connection represents strong and significant $(P$ value $<0.05, r>0.6)$ correlation. The size of each node is proportional to the number of connections, that is, degree. 


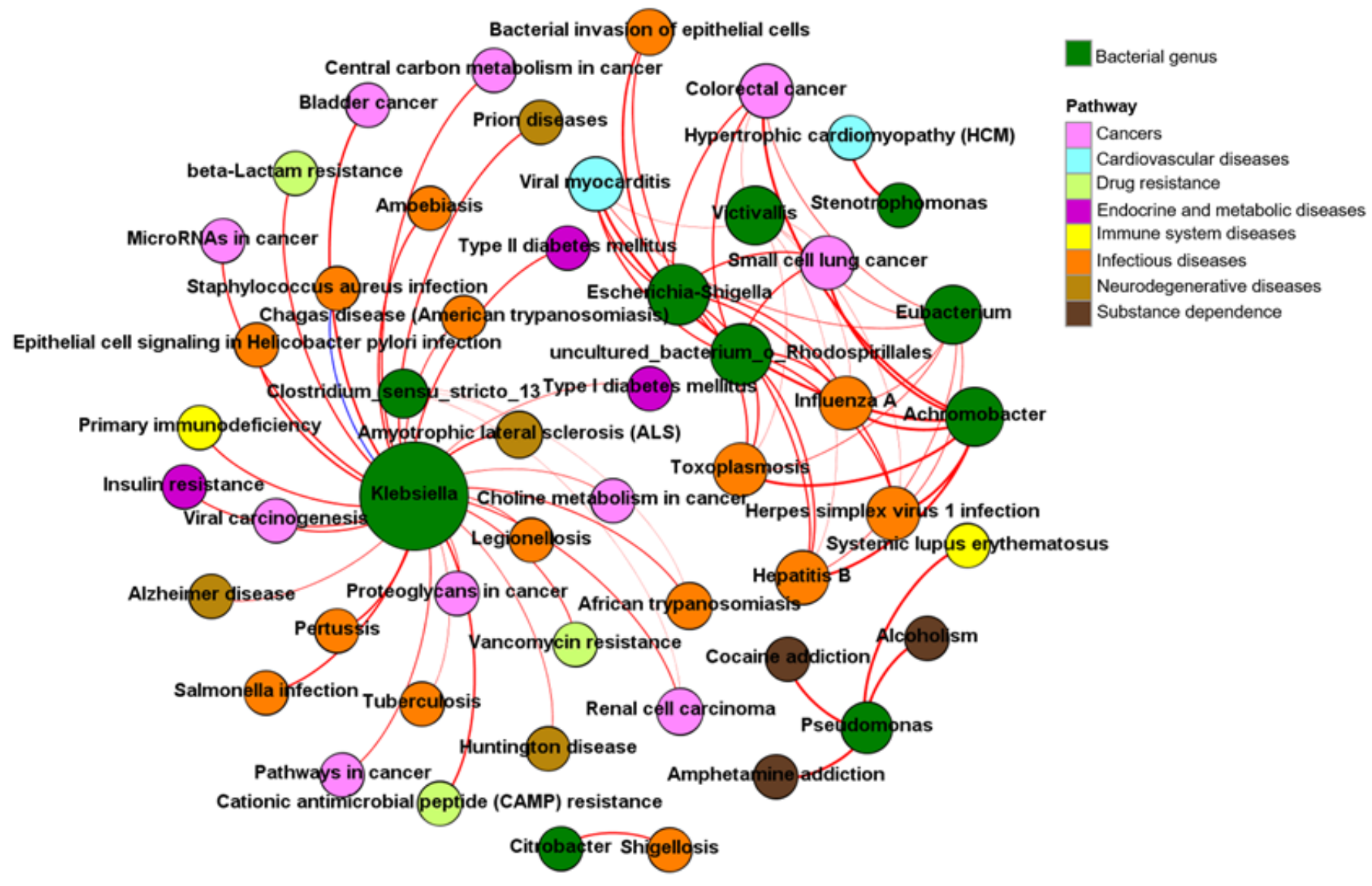

Figure 6

Network analysis revealing the co-occurrence patterns between microbial taxa and human disease-related pathways. The nodes in Network were colored according to human disease-related pathways types. The edges were colored according to positive (red) or negative (blue) correlation. A connection represents strong and significant $(P$ value $<0.05, r>0.6)$ correlation. The size of each node is proportional to the number of connections, that is, degree.

\section{Supplementary Files}

This is a list of supplementary files associated with this preprint. Click to download.

- Additionalfile2.xls

- Additionalfile1.doc 